\title{
Initial Concentration Normalized by
} Surface Area

National Cancer Institute

\section{Source}

National Cancer Institute. Initial Concentration Normalized by Surface Area. NCI

Thesaurus. Code $C 92385$.

Initial concentration divided by the surface area. Given only for bolus IV models. 